\title{
Fragilidad: ¿Cómo podemos detectarla?
}

\author{
$M^{a}$ Eugenia Pons Raventos*, Ana Rebollo Rubio**, José Vicente Jiménez Ternero** \\ *Fresenius Medical Care Services Andalucía, ** UGC Nefrología Hospital Regional Universitario de Málaga. España
}

\section{Sr. Director}

La fragilidad se puede definir como un estado fisiológico de mayor vulnerabilidad a los factores de estrés, que resultan de la disminución de las reservas fisiológicas o la desregulación de múltiples sistemas fisiológicos ${ }^{1}$. La opinión predominante es que una disminución fisiológica, asociada a la enfermedad o la edad, que individualmente no alcanza importancia clínica, al afectar a múltiples sistemas y tener un carácter acumulativo, puede ser detectado como fragilidad ${ }^{2}$. La fragilidad es señalada por distintos autores como un fuerte predictor de discapacidad, hospitalización, caídas, pérdida de la movilidad y enfermedad cardiovascular ${ }^{3}$, siendo los individuos frágiles más vulnerables a los episodios adversos ${ }^{2}$.

\section{Criterios de fragilidad}

Diferentes estudios han aplicado diferentes criterios para definir la presencia de fragilidad. La mayoría incluyen medidas de rendimiento físico o funcionamiento aportadas por el sujeto, así como algunos indicadores de fatiga o agotamiento y algunos también incluyen medidas de comorbilidad y discapacidad ${ }^{2}$. La definición más utilizada, desarrollada y validada en el Cardiovascular Health Study por Fried y sus colegas $^{4}$, se basa en la presencia de al menos tres de los siguientes cinco criterios: más de 10 libras de pérdida de peso involuntaria durante más de 1 año, fuerza de prensión débil, agotamiento, velocidad de la marcha lenta y escasa actividad física ${ }^{2}$. La importancia de la velocidad de la marcha para predecir la presencia de fragilidad en adultos, ha sido demostrado en los estudios realizados por Studenski et al. y Viccaro el al. ${ }^{5,6}$, siendo una herramienta útil para identificar a sujetos que estén en alto riesgo de deterioro de su salud y deterioro funcional.

La fragilidad es el paso previo a la discapacidad. La importancia de este concepto se centra en que en ocasiones este estado es susceptible de intervención. Y así, interviniendo en lo que es modificable o preveni- ble en los ancianos y pacientes frágiles, disminuiremos o retrasaremos la situación de discapacidad.

Las actividades instrumentales de la vida diaria son las que primero se ven afectadas. La necesidad de ayuda para estas actividades, es un indicador temprano de deterioro funcional, con implicaciones importantes para la calidad de vida de los individuos, y además ha demostrado solaparse con la presencia de fragilidad ${ }^{4,7}$.

El Ministerio de Sanidad y Política Social, en colaboración con el Instituto de Salud Carlos III, organismo autónomo del Ministerio de Ciencia e Innovación y la Fundación Progreso y Salud de Andalucía en el año 20098, ante la falta de consenso en la terminología y la escasa evaluación de herramientas para el diagnóstico de fragilidad del anciano, llevó a cabo un estudio, mediante el método Delphy (método basado en la opinión de un grupo de expertos) con el objetivo de definir el concepto de fragilidad en el anciano, mostrar herramientas y/o marcadores con los que obtener criterios diagnósticos y analizar las estrategias en el manejo de ancianos frágiles. Las conclusiones a las que llegaron este grupo de expertos fueron las siguientes:

- El 70,6\% de los autores de los estudios incluidos no crearon su propio concepto de fragilidad, utilizando la definición de otro autor y en ocasiones la definición de varios autores en un mismo artículo.

- Desde el punto de vista de la práctica clínica, se pueden clasificar las definiciones en dos grandes grupos: Factores Biomédicos, Fried et al. $2001^{4}$ 0 definiciones más holísticas donde se incluyen factores psicosociales y ambientales, Rockwood $1994^{9}$.

- Para la mayoría de los autores anciano frágil es igual a anciano vulnerable, aunque algunos autores asemejaron fragilidad a incapacidad, la mayoría consideró incapacidad como la pérdida de una función y la fragilidad el riesgo de perder dicha función. 
- Observaron variabilidad en los indicadores/marcadores utilizados para identificar la fragilidad y en ningún caso hallaron evidencia de que alguno de ellos fuese causa suficiente por sí misma que permita el diagnóstico de fragilidad en el anciano.

- Y lo más importante, no existe una herramienta validada para identificar la fragilidad, aunque encontraron la posibilidad de agrupar en 16 categorías todas la variables o posibles predictores. Son los siguientes: caídas, incontinencia, polimedicación, autopercepción de salud, actividades de la vida diaria, hospitalización, patologías, deterioro cognitivo, estado nutricional, úlceras por presión, parámetros clínicos, trastornos de la movilidad, disminución sensorial, trastornos del ánimo, edad y características sociodemográficas y ambientales.

- No se localizaron evidencias de ningún elemento individual en el sistema sanitario efectivo por sí mismo.

Por otro lado, existió consenso por el total de expertos en que existen unas variables predictoras de fragilidad por sí mismas.

No existe un estándar de oro para establecer la fragilidad, parece que el modelo de Fried es el más aceptado y usado en múltiples estudios que valoran fragilidad ${ }^{10-12}$. Actualmente, en la definición de fragilidad tiene mayor relevancia la aparición conjunta de varias características y la velocidad de instauración, que actúa a su vez como factores determinantes en la evolución y peor pronóstico de estas personas.

La labor de detección y asistencia del paciente frágil queda relegada casi exclusivamente a los profesionales del ámbito de la atención primaria y las enfermeras gestoras de casos hospitalarias. Es importante resaltar la importancia, para coordinar la labor de atender al anciano frágil, de disponer de una historia clínica unificada y de una buena comunicación interniveles de salud. Además de señalar que debido a la sobrecarga asistencial y burocrática en la que nos encontramos actualmente, hace más y más difícil poder atender de forma óptima a estos pacientes. La detección de ancianos frágiles no debe quedar relegada al ámbito de la atención primaria, siendo necesaria desarrollarla también en el ámbito hospitalario. En el hospital no debe limitarse solamente a aquellos con dependencia establecida o presencia de síndromes geriátricos, sino de forma más general a cualquiera con riesgo de deterioro funcional, lo que incluiría a los anteriores $\mathrm{y}$, además, a aquellos con un deterioro fun- cional más leve, quizás preclínico, que también tienen riesgo de deterioro funcional y que, si no es evaluado, podría pasar inadvertido ${ }^{13}$. Un estudio realizado en nuestro país sobre 379 pacientes, concluye que el deterioro funcional es frecuente durante la hospitalización en los pacientes frágiles, y que son, determinados factores asociados a la práctica clínica y por tanto modificables, los que se asocian a ese deterioro ${ }^{14}$. Coincide con otro estudio sobre pacientes hospitalizados que concluye que la hospitalización conlleva un deterioro importante en la capacidad funcional de los pacientes ancianos ${ }^{15}$.

\section{Instrumentos para medir fragilidad}

El Documento de consenso sobre prevención de fragilidad y caídas en la persona mayor, elaborado por el Ministerio de Sanidad, servicios Sociales e Igualdad en 2014, nos propone dos escalas para medir fragilidad:

- La herramienta "Instrumento de fragilidad para la atención primaria de la encuesta de salud, envejecimiento y Jubilación en Europa: SHARE-FI", validada para población española en 2011, basada en los criterios de Fried y Waltson ${ }^{4}$ aplicable en el ámbito de la atención primaria para pacientes no residentes en instituciones. A través de la web (http://www. biomedcentral.com/1471-2318/10/57), se encuentran calculadoras de acceso gratuito diferenciadas por sexo, y la versión española disponible en https://sites.google.com/a/tcd.ie/share-frailty-instrument-calculators/.

- Escala Frail ${ }^{16}$, de fácil aplicación, compuesta por 5 items (fatigabilidad, resistencia, deambulación, comorbilidad y pérdida de peso) en la que cada respuesta afirmativa es valorada con 1 punto, se considera fragilidad puntuaciones $\geq$ a 3 y prefragilidad 1 o 2 puntos. Aún no validada en población española.

- Existen otras escalas propuestas por otros autores como son la Tilburg Frailty Indicator ${ }^{17}$, la Groningen Frailty Indicator ${ }^{18}$ pero ninguna de ellas cuenta con validación para población española.

Recientemente en nuestro país se ha desarrollado una escala para medir las características biológicas de la fragilidad, The Frailty Trait Scale (FTS) ${ }^{19}$, basada en el modelo de fragilidad de Fried, aunque también incorpora del marco propuesto por Rockwood. Esta consta de 12 items y se ha aplicado a una muestra de 1972 hombres y mujeres mayores de 65 años. Identifica 7 dimensiones de fragilidad: balance energético-nutrición, actividad física, sistema nervioso, sistema vascular, fuerza, resistencia y velocidad de la 
marcha. Fue comparado con dos modelos para medir fragilidad bien establecidos: el fenotipo de fragilidad propuesto por Fried et al, y el índice de fragilidad (FI) propuesto por Rockwood y Mitnitski. Además de las cinco dimensiones propuestas por Fried et al, incluye otras dimensiones como son el estado cognitivo, nutricional y vascular. EI FTS tiene un valor predictivo que es ligeramente mejor que el modelo de Fried para la mortalidad en personas mayores de 80 años, y para las hospitalizaciones en menores de 80 años.

Recibido: 07 marzo 16

Revisado: 18 marzo 16

Modificado: 28 marzo 16

Aceptado: 11 marzo 16

\section{Bibliografía}

1. Fried LP, Ferrucci L, Darer J, Williamson JD, Anderson G. Untangling the concepts of disability, frailty, and comorbidity: implications for improved targeting and care. J Gerontol A Biol Sci Med Sci. 2004; 59(3):255-263.

2. Johansen KL, Delgado C, Bao Y, Kurella Tamura M. Frailty and dialysis initiation. Semin Dial. 2013; 26(6):690-696.

3. Romero Rizos L, Abizanda Soler P. Fragilidad como predictor de episodios adversos en estudios epidemiológicos: revisión de la literatura. Rev Esp Geriatr Gerontol. 2013; 48(6):285-289.

4. Fried LP, Tangen CM, Walston J, Newman AB, Hirsch C, Gottdiener J, et al. Frailty in older adults: evidence for a phenotype. J Gerontol A Biol Sci Med Sci. 2001; 56(3):M146-156.

5. Studenski S, Perera S, Patel K, Rosano C, Faulkner $\mathrm{K}$, Inzitari M, et al. Gait speed and survival in older adults. JAMA. 2011; 305(1):50-58.

6. Viccaro LJ, Perera S, Studenski SA. Is timed up and go better than gait speed in predicting health, function, and falls in older adults? J Am Geriatr Soc. $2011 ;$ 59(5):887-892.

7. Kutner NG, Zhang R, Allman RM, Bowling CB. Correlates of $A D L$ difficulty in a large hemodialysis cohort. Hemodial Int. 2014; 18(1):70-77.

8. Carlos-Gil AM, Martínez-Pecino F, Molina-Linde JM, Villegas-Portero R, Aguilar-García J, GarcíaAlegría J, et al. Desarrollo de criterios, indicadores de complejidad y estrategias de manejo en fragilidad. Sevilla: Agencia de Evaluación de Tecnologías Sanitarias de Andalucía; 2009.

9. Rockwood K, Fox RA, Stolee P, Robertson D, Beattie BL. Frailty in elderly people: an evolving concept. CMAJ. 1994; 150(4):489-495.

10. Jürschik $P$, Escobar MÁ, Nuin C, Botigué T. Criterios de fragilidad del adulto mayor. Estudio piloto. Aten Primaria. 2011; 43(4):190-196. 
11. González-Vaca J, de la Rica-Escuín M, SilvaIglesias $M$, Arjonilla-García MD, VarelaPérez $\mathrm{R}$, Oliver-Carbonell $\mathrm{JL}$, et al. Frailty in Institutionalized older adults from Albacete. The FINAL Study: rationale, design, methodology, prevalence and attributes. Maturitas. 2014; 77(1):78-84.

12. Rothman MD, Leo-Summers L, GillTM. Prognostic significance of potential frailty criteria. J Am Geriatr Soc. 2008; 56(12):2211-2216.

13. Baztán-Cortés JJ, González-Montalvo JI, SolanoJaurrieta JJ, Hornillos-Calvo M. Atención sanitaria al anciano frágil: de la teoría a la evidencia científica. Med Clin (Barc). 2000; 115(18):704-717.

14. Vidán-Astiz MT, Sánchez-García E, AlonsoArmesto M, Montero-Errasquin B, Martínez-dela-Casa A, Ortiz FJ, et al. Deterioro funcional durante la hospitalización en ancianos. Beneficios del ingreso en el servicio de geriatría. Rev Esp Geriatr Gerontol. 2008; 43(3):133-138.

15. Mañas MD, Marchán E, Conde C, Sánchez S, Sánchez-Maroto T, Molina MC. Deterioro de la capacidad funcional en pacientes ancianos ingresados en un Servicio de Medicina Interna. An Med Interna. 2005; 22(3):130-132.
16. Morley JE, Malmstrom TK, Miller DK. A simple frailty questionnaire (FRAIL) predicts outcomes in middle aged African Americans. J Nutr Health Aging. 2012; 16(7):601-608.

17. Gobbens RJJ, van Assen MALM, Luijkx KG, Wijnen-Sponselee MT, Schols JMGA. The Tilburg Frailty Indicator: psychometric properties. J Am Med Dir Assoc. 2010; 11(5):344-355.

18. Peters LL, Boter H, Buskens E, Slaets JPJ. Measurement properties of the Groningen Frailty Indicator in home-dwelling and institutionalized elderly people. J Am Med Dir Assoc. 2012; 13(6):546-551.

19. García-García FJ, Carcaillon L, FernandezTresguerres J, Alfaro A, Larrion JL, Castillo C, et al. A new operational definition of frailty: the Frailty Trait Scale. J Am Med Dir Assoc. 2014; 15(5):371.e7-371.e13. 